\title{
Observations on dentine hypersensitivity in general dental practices in the United Arab Emirates
}

Hasanain Al-Khafaji ${ }^{1}$

Correspondence: Dr. Hasanain Al-Khafaji

Email: halkhafaji@sharjah.ac.ae
'Department of General and Specialized Dental Practice, College of Dentistry, University of Sharjah, Sharjah, United Arab Emirates

\section{ABSTRACT}

Dentine hypersensitivity is a common clinical finding with a wide variation in prevalence values and etiological factors. The objective of this cross-sectional study was to investigate the prevalence and some etiological factors of dentine hypersensitivity of Emirati patients visiting general dental clinics in the United Arab Emirates (UAE) over a period of three calendar months. Materials and Methods: Six general dental practitioners examined 204 Emirati patients over a period of three calendar months and patients who had dentine hypersensitivity diagnosed were questioned further about their smoking habits and the frequency, severity, and duration of their pain. Furthermore, cervical tooth surface loss was noted. Results: A total of 55 patients were diagnosed as having dentine hypersensitivity, giving a prevalence figure of $27 \%$. The most common teeth affected were the lower anterior teeth. Conclusions: The prevalence of dentine hypersensitivity in Emirati patients visiting a general dental clinic in the UAE was $27 \%$. The most common etiology appeared to be the loss of cervical tooth surface structure.

Key words: Dental pain, dentine hypersensitivity, etiology of dentine hypersensitivity, smoking habits

\section{INTRODUCTION}

Dentine hypersensitivity also known as root sensitivity has been defined as "a short, sharp pain arising from exposed dentine, in response to stimuli, typically thermal, evaporative, tactile, osmotic or chemical, and which cannot be ascribed to any other form of dental defect or pathology." [1,2] Other terms that have been used to describe this condition include dentine sensitivity, root sensitivity, cervical sensitivity, and hypersensitivity. All of these terms have one thing in common; however, they are all interpreted as pain.

Dentine hypersensitivity is a common problem found in many adult populations with prevalence figures reported to range between $4 \%$ and $67.7 \% .^{[2]}$

This wide variation in prevalence may be due to a number of factors, including the population studied, and/or the methodology used (e.g., questionnaire vs. clinical examination). Patient questionnaire based surveys are thought to overstate true prevalence. ${ }^{[3]}$ The majority of the previous investigations of dentine hypersensitivity [Table 1] have included subspecialty referral populations and have been carried out mostly on non-Arab populations. ${ }^{[3-16]}$

The objective of this cross-sectional study was to investigate the prevalence and explore factors that may pre-dispose Emirati patients visiting general dental clinics in the United Arab Emirates (UAE) to dentine hypersensitivity.

\section{MATERIALS AND METHODS}

Six general dental practitioners with at least 6 years of practical experience were recruited to participate in this study randomly and all of the participants

\footnotetext{
How to cite this article: Al-Khafaji $\mathrm{H}$. Observations on dentine hypersensitivity in general dental practices in the United Arab Emirates. Eur J Dent 2013;7:389-94. 


\begin{tabular}{|c|c|c|c|c|c|}
\hline Authors & Country & Setting & Study type & $n$ & Prevalence (\%) \\
\hline Jensen $(1964)^{[4]}$ & USA & University & Clinical & 3000 & 30 \\
\hline Flynn et al. $(1985)^{[6]}$ & Switzerland & Practice & Clinical & 351 & 15 \\
\hline Orchardson and Collins $(1987)^{[7]}$ & UK & University & Clinical & 369 & 18 \\
\hline Fischer et at. $(1992)^{[8]}$ & UK & University & Clinical & 109 & 74 \\
\hline Murray and Roberts $(1994)^{[9]}$ & Brazil & University & Clinical & 635 & 17 \\
\hline Murray and Roberts (1994) & Indonesia & Not stated & Questionnaire & 1000 & 27 \\
\hline Murray and Roberts $(1994)^{[9]}$ & USA & Not stated & Questionnaire & 1000 & 18 \\
\hline Murray and Roberts $(1994)^{[9]}$ & Japan & Not stated & Questionnaire & 1000 & 16 \\
\hline Murray and Roberts $(1994)^{[9]}$ & France & Not stated & Questionnaire & 1000 & 14 \\
\hline Murray and Roberts (1994) ${ }^{[9]}$ & Germany & Not stated & Questionnaire & 1000 & 13 \\
\hline Chen et al. $(1994)^{*}$ & Australia & Not stated & Questionnaire & 1000 & 13 \\
\hline Chabanski et al. $(1997)^{[11]}$ & USA & University & Clinical & 184 & 50 \\
\hline Irwin and McCusker (1997) $)^{[12]}$ & UK & University & Clinical & 51 & 73 \\
\hline Liu, Lan and Hsieh (1998)[13] & UK & Practice & Questionnaire & 250 & 57 \\
\hline Ress $(2000)^{[3]}$ & Taiwan & University & Clinical & 780 & 32 \\
\hline Tanni and Twartani $(2002)^{[14]}$ & UK & Practice & Clinical & 3593 & 4 \\
\hline Clayton et al. (2002) ${ }^{[15]}$ & Saudi Arabia & University & Clinical & 295 & $42-60$ \\
\hline Ress and Addy $(2002)^{[16]}$ & UK & Air force & Questionnaire & 228 & 50 \\
\hline Ress et al. $(2003)^{[17]}$ & UK & Practice & Clinical & 4841 & 4,1 \\
\hline
\end{tabular}

completed the study. The study ran from November $1 / 2008$ to January $31 / 2009$. Prior to the start of the study, the practitioners met with the author to finalize details of the study protocol. The study protocol emphasized that in order to make the diagnosis of dentine hypersensitivity, other pathologies, such as caries, enamel cracks, and restored teeth must be ruled out. To be considered for the study, the patients had to be Emirati be new patients to the practice or pre-existing patients presenting with a new complaint. The patients were given information about dentine hypersensitivity and all of the patients seen by each dentist during the trial period were screened for sensitive teeth prior to completion of the questionnaire related to dentine hypersensitivity. If the patient then recorded a positive response on the questionnaire, the diagnosis of dentine hypersensitivity was confirmed using a blast of air from a triple syringe. Tactile sensitivity using a probe applied to the cervical region was not assessed as it has been reported that there is no difference in the subjective response to tactile and evaporative stimuli in the detection of dentine hypersensitivity ${ }^{[10]}$ Where a diagnosis of dentine hypersensitivity was made, a study form was completed. This form included details of the patient's age, gender, occupation, and smoking habits. Furthermore, included was the history of dentine hypersensitivity, such as the teeth affected and the frequency, severity, and the duration of the complaint. Details regarding any previous treatment and any factors thought to have initiated the sensitivity were sought. In addition to this, the clinicians were asked to measure any buccal gingival recession associated with these sensitive teeth. Measurements were made using a $1 \mathrm{~mm}$ graduated periodontal probe from the amelocemental junction to the free gingival margin. The participating dentists were also asked to record the total number of patients seen during the trial period and the methods that the dentists employed to manage the sensitivity.

The relationship between dentine hypersensitivity and social class was examined using the Registrar General's Classification of Occupations as used by Bradnock et al. in the UK Adult Dental Health Survey ${ }^{[17]}$ This divides occupations into a series of six groups using the following classifications:

I: Professional (e.g., doctor, dentist, lawyer)

II: Managerial and lower professional (e.g., manager, nurse, school teacher)

IIIN: Skilled, non-manual (e.g., clerk, cashier)

IIIM: Skilled, manual (e.g., carpenter, bricklayer, coal face worker)

IV: Semi-skilled, manual (e.g., postman, agricultural worker)

V: Unskilled, manual (e.g., porter, ticket collector, general laborer) ${ }^{[17]}$

\section{RESULTS}

Two hundred and four Emirati patients were seen by the six general dental practitioners who were involved in the study (128 male patients and 76 female 
patients). A total of 55 patients were diagnosed as having dentine hypersensitivity, giving an overall prevalence figure for dentine hypersensitivity of $27 \%$. Nearly, $29 \%$ of the male patients in this study were found to have hypersensitivity, versus $22 \%$ of the female patients examined [Table 2].

A histogram showing the age distribution of the patients with hypersensitive dentine is given in Figure 1. This figure makes it clear that the highest numbers of patients with dentine hypersensitivity were in the age range of 20-29 years.

The distribution by tooth type is given in Figure 2 and shows that the lower anterior teeth were most commonly affected by dentine hypersensitivity.

The mean number of sensitive teeth per patient by age group is given in Figure 3. This demonstrates that the number of sensitive teeth in this sample peaked at 8.2 in the 50-59 year age group.

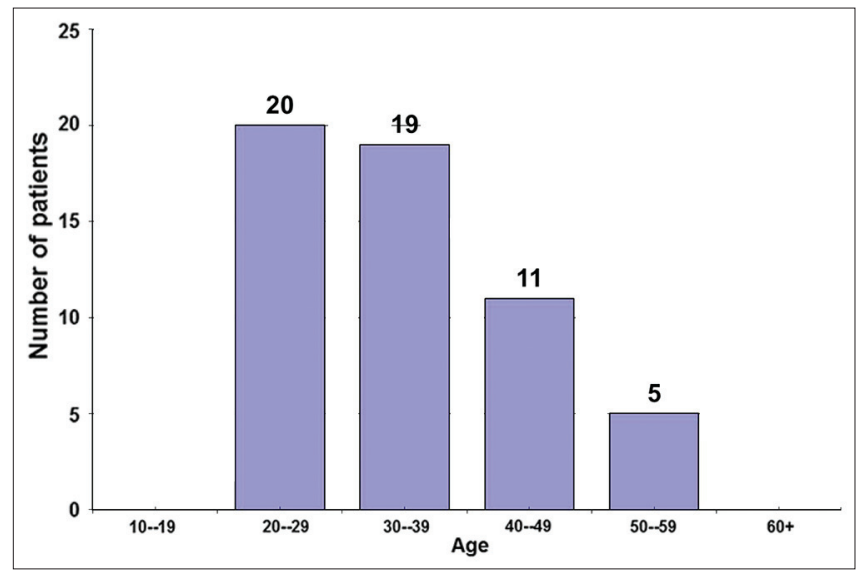

Figure 1: The age distribution of patients with hypersensitive teeth

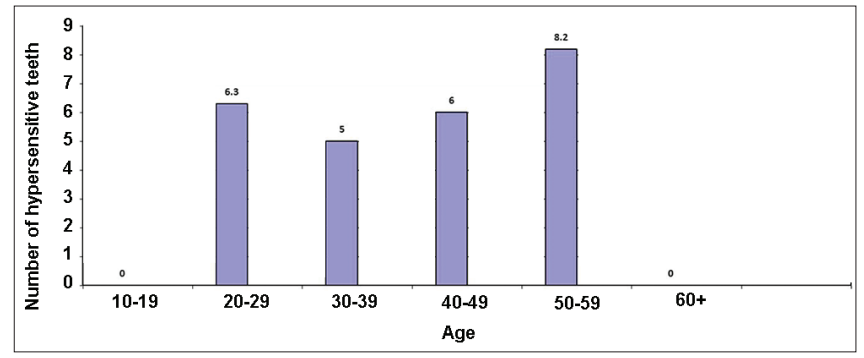

Figure 3: The mean number of hypersensitive teeth per patient
Figure 4 shows the relationship between dentine hypersensitivity and smoking, and the distribution of dentine hypersensitivity between male and female smokers and non-smokers. The difference between the smokers and non-smokers was $5 \%$.

This study found that in $68 \%$ of cases, the etiology of dentine hypersensitivity was due to the loss of cervical tooth surface structure (abrasion, attrition, erosion, and abfraction). The study also found that $15 \%$ of patients with sensitive teeth suffer from gingival recession.

Sixty four percent of the patients with hypersensitive teeth reported experiencing pain "occasionally" in response to stimuli such as cold or hot drinks. Only $2 \%$ reported having pain "all the time," while $34 \%$ encountered pain "most of the time". When

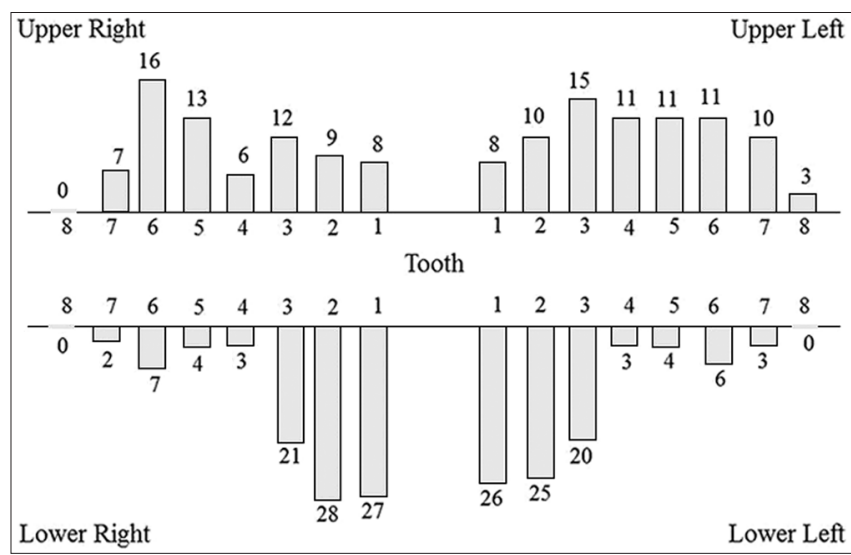

Figure 2: Frequency of dentine hypersensitivity by tooth type and quadrant

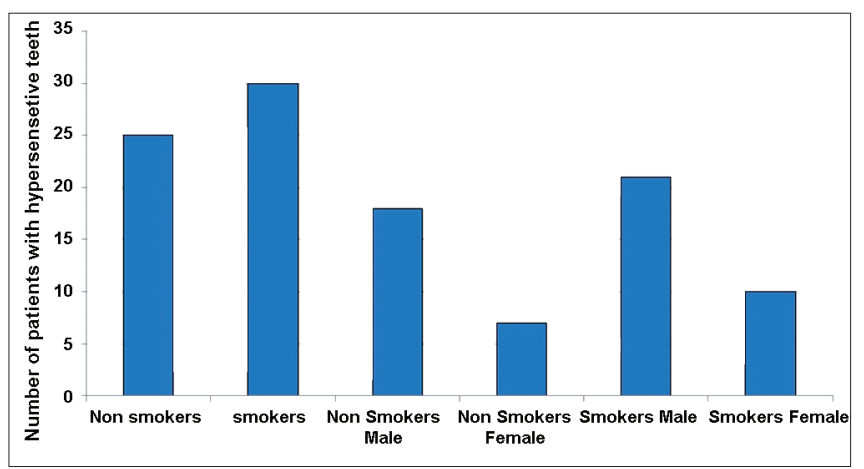

Figure 4: The frequency of hypersensitivity for male and females according to smoking habit

Table 2: Smoker patients reporting on dentine hypersensitivity of teeth

\begin{tabular}{lcccccc} 
Sex & $\boldsymbol{N}$ & $\begin{array}{c}\text { Hypersensitivity } \\
\text { absent }\end{array}$ & $\begin{array}{c}\text { Hypersensitivity } \\
\text { present }\end{array}$ & $\begin{array}{c}\text { Nonsmoker patients } \\
\text { with hypersensitivity } \boldsymbol{N}\end{array}$ & $\begin{array}{c}\text { Smoker patients with } \\
\text { hypersensitivity } \boldsymbol{N}\end{array}$ & $\begin{array}{c}\text { Hypersensitivity } \\
\text { (\%) }\end{array}$ \\
\hline Male & 128 & 90 & 38 & 18 & 21 & 69 \\
Female & 76 & 59 & 17 & 7 & 10 & 31 \\
Total & 204 & 149 & 55 & 25 & 30 & 27 \\
\hline
\end{tabular}


those patients were asked about the severity of hypersensitivity, 59\% had a "slight to moderate concern" while only $11 \%$ had a "severe concern". Approximately, $36 \%$ of the patients reported that they "never" avoided the sensitive area while $57 \%$ claimed that they avoided it "sometimes".

Approximately, $14 \%$ of the patients claimed that dentine hypersensitivity was present for "1-6 days," while $34 \%$ reported duration of "1-4 weeks." Approximately, $41 \%$ stated that their discomfort lasted for "1-12 months," while $11 \%$ claimed that it had lasted more than "1 year" [Table 3].

About $34 \%$ of the patients had received professional treatment for their hypersensitive teeth, and 55\% reported using desensitizing dentifrice.

Figure 5 shows the relationship between dentine hypersensitivity and social class. It was found that of the patients with sensitivity, $71 \%$ could be classified into the first three groups I, II and IIIN.

\section{DISCUSSION}

We found that the prevalence of dentine hypersensitivity among a sample of Emirati patients attending six general dental clinics in the UAE was $27 \%$. However, in comparing these results to those of other studies, caution is needed.

Firstly, some of the previous investigations have used patient questionnaires alone to assess prevalence. This methodology is likely to overestimate the prevalence of dentine hypersensitivity as the sensitivity reported might be due to other pathologies such as caries or cracked cusps, which could easily be detected by clinical examination. ${ }^{[15]}$ Secondly, the wide range of individual prevalence figures may suggest that any differences were due to factors related to individual examiners rather than their patients.

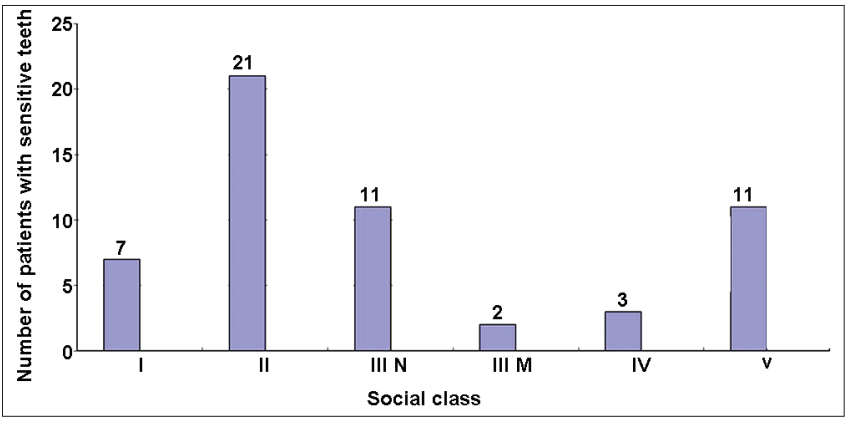

Figure 5: Patients with hypersensitive teeth classified by social class
Finally, variations are likely due to differences in the populations studied. ${ }^{[18]}$

Dentin is naturally sensitive owing to its close structural and functional relationship with the dental pulp. ${ }^{[19]}$ This inherent sensitivity usually is not a problem because normally other tissues cover the dentin.

Dentinal pain is thought to be mediated by a hydrodynamic mechanism. According to this hypothesis, a pain-provoking stimulus applied to dentin increases the flow of dentinal tubular fluid. In turn, this mechanically activates the nerves situated at the inner ends of the tubules or in the

\begin{tabular}{|c|c|}
\hline The questionnaire & $\%$ \\
\hline \multicolumn{2}{|l|}{ Etiology of DH } \\
\hline Gingival recession & 15 \\
\hline Erosion & 16 \\
\hline Abrasion & 27 \\
\hline Attrition & 20 \\
\hline Abfraction & 5 \\
\hline Bleaching & 7 \\
\hline Periodontal therapy & 5 \\
\hline Aging & 2 \\
\hline Medication & 0 \\
\hline Other & 2 \\
\hline \multicolumn{2}{|l|}{ Frequency of $\mathrm{DH}$} \\
\hline Occasionally & 64 \\
\hline Most of the time & 34 \\
\hline All of the time & 2 \\
\hline \multicolumn{2}{|l|}{ Severity of $\mathrm{DH}$} \\
\hline No concern & 30 \\
\hline Slight to moderate concern & 59 \\
\hline Severe concern & 11 \\
\hline \multicolumn{2}{|l|}{ Avoidance of sensitive teeth } \\
\hline Never & 36 \\
\hline Some times & 57 \\
\hline Most of the time & 7 \\
\hline \multicolumn{2}{|l|}{ Duration of $\mathrm{DH}$} \\
\hline 1-3 Day (s) & 7 \\
\hline 4-6 Days & 7 \\
\hline 1-4 week (s) & 34 \\
\hline $1-12$ month (s) & 41 \\
\hline$>1$ year & 11 \\
\hline \multicolumn{2}{|l|}{ Professional treatment of $\mathrm{DH}$} \\
\hline Yes & 44 \\
\hline No & 66 \\
\hline \multicolumn{2}{|l|}{ Use of desensitizing dentifrice } \\
\hline Yes & 55 \\
\hline No & 45 \\
\hline
\end{tabular}


outer layers of the pulp. ${ }^{[18]}$ Evidence suggests that patients complain from dentine hypersensitivity when dentine is exposed and the dentinal tubule system is open to the oral cavity. The process needed to localize lesions of dentine hypersensitivity includes loss of enamel and/or gingival recession. This has led some authors to suggest dentine hypersensitivity is a tooth wear phenomenon-while acknowledging that much remains unknown or unproven about the etiology of this condition. ${ }^{[20]}$

However; not all exposed dentin is sensitive. Sensitivity is thought to occur when the smear layer or tubular plugs are removed, opening the outer ends of dentinal tubules. ${ }^{[18]}$ Microscopic examination reveals that patent dentinal tubules are more numerous and wider in hypersensitive dentin than in non-sensitive dentin. ${ }^{[16,21]}$

Several investigators have reported the age distribution of dentine hypersensitivity. Orchardson and Collins reported a peak prevalence between 20 years and 25 years; ${ }^{[7]}$ Graf and Galasse between 25 years and 29 years; ${ }^{[5]}$ Addy observed peak prevalence between 20 years and 40 years; ${ }^{[22]}$ and Fischer et al. between 40 years and 49 years. ${ }^{\left[{ }^{[8]}\right.}$ In our study, dentine hypersensitivity was the greatest in the 20-39 years age group, with a peak prevalence in the 20-29 years age group $(n=20)$ and slightly lower in the 30-39 years age group $(n=19)$.

This age distribution of dentine hypersensitivity appears to be in closest agreement with the data reported by Orchardson and Collins; ${ }^{[7]}$ Graf and Galasse, ${ }^{[5]}$ and Addy. ${ }^{[22]}$

The probable reason for the observed reduction in dentine hypersensitivity with increasing age of patients may be due to the laying down of secondary or tertiary dentine. ${ }^{[23]}$ However, the high prevalence in younger age group patients may result from their cultural attitudes towards oral health and their home care habits, in addition to the increasing numbers of young patients who get restorative treatments or teeth whitening with vital bleaching techniques in the UAE. This may be similar to the findings reported by Haywood. ${ }^{[24]}$ All of the teeth in this study exhibiting dentine hypersensitivity also had some degree of gingival recession. Most teeth had at least 1-3 mm of gingival recession $(n=15)$, which is similar to the average recession of $2.5 \mathrm{~mm}$ reported by Addy et al. in their sample of sensitive teeth. ${ }^{[25]}$
The teeth most often affected by dentine hypersensitivity were the lower incisors, followed by the premolars, then the canines, and then the upper molars. This distribution is reminiscent of the reports of Rees et al. ${ }^{[16]}$ Taani and Awartani studies, ${ }^{[13]}$ but dissimilar to Rees and Addy, ${ }^{[15]}$ and Rees, ${ }^{[3]}$ and earlier studies that reported the upper premolars most affected. Since the lower incisors are the teeth most affected by calculus accumulation followed by non-surgical periodontal therapy and because of the esthetic impact of these teeth, the lower incisors are more likely to be retained, even when severely compromised. ${ }^{[26]}$

The mean number of sensitive teeth per patient peaked at about 8 in the 50-59 year group, which is higher than the values reported in several of the studies mentioned above. ${ }^{[2,27]}$

It has been hypothesized that dentine hypersensitivity might be more common among smokers, as they are more prone to gingival recession. However, the data from this study found no association between dentine hypersensitivity and smoking. A recent report by Müller et al. suggested that smokers are not at risk for gingival recession, ${ }^{[26]}$ but other studies, including those of Al-Wahadni and Linden, ${ }^{[28]}$ and Rees and Addy, ${ }^{[15]}$ have found more gingival recession and sensitivity among smokers.

The previous studies (Fischer et al. ${ }^{[8]}$ Orchardson and Collins; ${ }^{[7]}$ Addy et al. ${ }^{[25]}$ Flynn et al..$^{[6]}$ Cunha et al. ${ }^{[29]}$ Oyama and Matsumoto; ${ }^{[30]}$ Taani and Awartani; ${ }^{[31]}$ Rees; ${ }^{[3]}$ Rees and Addy $\left.{ }^{[15]}\right)$ reported a higher incidence of dentine hypersensitivity in females than in males. In this study, the ratio of females to males with hypersensitivity was 1.3:1; this difference is not likely to be statistically significant.

About $11 \%$ of patients in the current study reported avoiding hypersensitive teeth most of the time. This figure is similar to that reported by Taani and Awartani. ${ }^{[31]}$ Approximately, 34\% of patients in this study were treated for dentine hypersensitivity by dentists, and $55 \%$ had tried treatment with desensitizing dentifrice. These figures are higher than those reported by Taani and Awartani, ${ }^{[31]}$ Liu et al. ${ }^{[12]}$ and Fischer et al. ${ }^{[8]}$

It is the author's clinical impression, supported by some data, (Absi et al.), ${ }^{[32]}$ that dentine hypersensitivity is more prevalent among patients who have good oral hygiene practices as tends to be the case in higher socioeconomic groups. To investigate this further, the 
patients with dentine hypersensitivity were divided into social groups using the Registrar General's Classification of Occupations as used in the recent UK Adult Dental Health Survey. ${ }^{[33,34]}$ This demonstrated that dentine hypersensitivity was more prevalent in the higher social groups, with $71 \%$ of the sensitive teeth being found in the top three social groups I, II and IIIN. However, this data must also be interpreted with a certain amount of caution as sampling bias due to dental clinic attendance is very likely.

\section{CONCLUSIONS}

The findings of the present study have revealed that the prevalence of dentine hypersensitivity in Emirati patients visiting a general dental clinic in the UAE was $27 \%$. The prevalence of dentine hypersensitivity in this sample was high in the $3^{\text {rd }}$ and $4^{\text {th }}$ decades of age. On the same time, the prevalence of dentine hypersensitivity was more prevalent in the high social groups. Some differences in our findings when compared to other studies may reflect variations in study methodology as well as national differences in attitudes toward oral health and home care procedures. Improved patient education in dental hygiene and preventive measures might reduce these figures.

\section{REFERENCES}

1. Addy M. Dentine Hypersensitivity: New perspectives on an old problem. Int Dent J 2002;52:367-75.

2. Bartold PM. Dentinal hypersensitivity: A review. Aust Dent J 2006;51:212-8; quiz 276.

3. Rees JS. The prevalence of dentine hypersensitivity in general dental practice in the UK. J Clin Periodontol 2000;27:860-5.

4. Jensen AL. Hypersensitivity controlled by iontophoresis: Double blind clinical investigation. J Am Dent Assoc 1964;68:216-25.

5. Graf H, Galasse R. Morbidity, prevalence and intraoral distribution of hypersensitive teeth. J Dent Res 1977;56:162.

6. Flynn J, Galloway R, Orchardson R. The incidence of 'hypersensitive' teeth in the West of Scotland. J Dent 1985;13:230-6.

7. Orchardson R, Collins WJ. Clinical features of hypersensitive teeth. Br Dent J 1987;162:253-6.

8. Fischer C, Fischer RG, Wennberg A. Prevalence and distribution of cervical dentine hypersensitivity in a population in Rio de Janeiro, Brazil. J Dent 1992;20:272-6.

9. Murray LE, Roberts AJ. The prevalence of self reported hypersensitive teeth. Arch Oral Biol 1994;39:129.

10. Chabanski MB, Gillam DG, Bulman JS, Newman HN. Clinical evaluation of cervical dentine sensitivity in a population of patients referred to a specialist periodontology department: A pilot study. J Oral Rehabil 1997;24:666-72.

11. Irwin CR, McCusker P. Prevalence of dentine hypersensitivity in a general dental population. J Ir Dent Assoc 1997;43:7-9.

12. Liu HC, Lan WH, Hsieh CC. Prevalence and distribution of cervical dentin hypersensitivity in a population in Taipei, Taiwan. J Endod 1998;24:45-7.

13. Taani SD, Awartani F. Clinical evaluation of cervical dentin sensitivity (CDS) in patients attending general dental clinics (GDC) and periodontal specialty clinics (PSC). J Clin Periodontol
2002;29:118-22.

14. Clayton DR, McCarthy D, Gillam DG. A study of the prevalence and distribution of dentine sensitivity in a population of 17-58-year-old serving personnel on an RAF base in the Midlands. J Oral Rehabil 2002;29:14-23.

15. Rees JS, Addy M. A cross-sectional study of dentine hypersensitivity. J Clin Periodontol 2002;29:997-1003.

16. Rees JS, Jin LJ, Lam S, Kudanowska I, Vowles R. The prevalence of dentine hypersensitivity in a hospital clinic population in Hong Kong. J Dent 2003;31:453-61.

17. Bradnock G, White DA, Nuttall NM, Morris AJ, Treasure ET, Pine CM. Dental attitudes and behaviours in 1998 and implications for the future. Br Dent J 2001;190:228-32.

18. Orchardson R, Gillam DG. Managing dentin hypersensitivity. J Am Dent Assoc 2006;137:990-8.

19. Orchardson R, Cadden SW. An update on the physiology of the dentine-pulp complex. Dent Update 2001;28:200-6, 208-9.

20. Addy M. Tooth brushing, tooth wear and dentine hypersensitivity - Are they associated? Int Dent J 2005;55:261-7.

21. Yoshiyama M, Noiri Y, Ozaki K, Uchida A, Ishikawa Y, Ishida H. Transmission electron microscopic characterization of hypersensitive human radicular dentin. J Dent Res 1990;69:1293-7.

22. Addy M. Clinical aspects of dentine hypersensitivity. Proc Finn Dent Soc 1992;88:23-30

23. Brahmbhatt N, Bhavsar N, Sahayata V, Acharya A, Kshatriya P. A double blind controlled trial comparing three treatment modalities for dentin hypersensitivity. Med Oral Patol Oral Cir Bucal 2012;17:e483-90.

24. Haywood VB. Dentine hypersensitivity: Bleaching and restorative considerations for successful management. Int Dent J 2002;52:376-84.

25. Addy M, Mostafa P, Newcombe RG. Dentine hypersensitivity: The distribution of recession, sensitivity and plaque. J Dent 1987;15:242-8.

26. Müller HP, Stadermann S, Heinecke A. Gingival recession in smokers and non-smokers with minimal periodontal disease. J Clin Periodontol 2002;29:129-36.

27. Amarasena N, Spencer J, Ou Y, Brennan D. Dentine hypersensitivity-Australian dentists' perspective. Aust Dent J 2010;55:181-7.

28. Al-Wahadni A, Linden GJ. Dentine hypersensitivity in Jordanian dental attenders. A case control study. J Clin Periodontol 2002;29:688-93.

29. Cunha-Cruz J, Wataha JC, Heaton LJ, Rothen M, Sobieraj M, Scott J, et al. The prevalence of dentin hypersensitivity in general dental practices in the northwest United States. J Am Dent Assoc 2013:144:288-96.

30. Oyama T, Matsumoto K. A clinical and morphological study of cervical hypersensitivity. J Endod 1991;17:500-2.

31. Taani Q, Awartani F. Clinical evaluation of cervical dentin sensitivity (CDS) in patients attending general dental clinics (GDC) and periodontal specialty clinics (PSC). J Clin Periodontol 2002;29:118-122.

32. Absi EG, Addy M, Adams D. Dentine hypersensitivity. A study of the patency of dentinal tubules in sensitive and non-sensitive cervical dentine. J Clin Periodontol 1987;14:280-4.

33. Dummer PM, Addy M, Hicks R, Kingdon A, Shaw WC. The effect of social class on the prevalence of caries, plaque, gingivitis and pocketing in 11-12-year-old children in South Wales. J Dent 1987;15:185-90.

34. Bradnock G, White DA, Nuttall NM, Morris AJ, Treasure ET, Pine CM. Dental attitudes and behaviours in 1998 and implications for the future. Br Dent J 2001;190:228-32.

\begin{tabular}{|l|l|}
\hline \multicolumn{2}{|c|}{ Access this article online } \\
\hline Quick Response Code: & Website: \\
& www.eurjent.com \\
\cline { 2 - 3 } & \\
&
\end{tabular}

